\title{
Cabozantinib in Advanced Hepatocellular Carcinoma: Efficacy and Safety Data from an International Multicenter Real-Life Cohort
}

\author{
Fabian Finkelmeier ${ }^{a, b, c}$ Bernhard Scheiner ${ }^{d, e}$ Catherine Leyh ${ }^{f}$ Jan Best ${ }^{g}$ \\ Thorben Wilhelm Fründt ${ }^{\mathrm{h}}$ Carolin Czauderna ${ }^{\mathrm{i}, \mathrm{j}, \mathrm{k}}$ Alica Beutel' Dominik Bettinger ${ }^{\mathrm{m}, \mathrm{n}}$ \\ Johannes Weiß ${ }^{\circ}$ Tobias Meischl ${ }^{d, e}$ Fabian Kütting ${ }^{p}$ Dirk-Thomas Waldschmidt ${ }^{p}$ \\ Pompilia Radu ${ }^{q}$ Michael Schultheiß ${ }^{m}$ Kai-Henrik Peiffer ${ }^{a}$ Thomas J. Ettrich ${ }^{1}$ \\ Arndt Weinmann $^{\mathrm{i}, j}$ Henning Wege $^{\mathrm{h}}$ Marino Venerito ${ }^{\mathrm{g}}$ Jean-Francois Dufour ${ }^{\mathrm{q}}$ \\ Christian M. Lange ${ }^{f}$ Matthias Pinter ${ }^{d, e}$ Oliver Waidmann ${ }^{a, b}$
}

aDepartment of Gastroenterology, Hepatology and Endocrinology, University Hospital Frankfurt, Frankfurt,

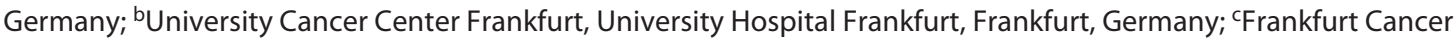
Institute, Goethe University Frankfurt/Main, Frankfurt, Germany; ${ }^{d}$ Division of Gastroenterology and Hepatology, Department of Internal Medicine III, Medical University of Vienna, Vienna, Austria; ' $L i v e r$ Cancer (HCC) Study Group Vienna, Medical University of Vienna, Vienna, Austria; 'Department of Gastroenterology and Hepatology,

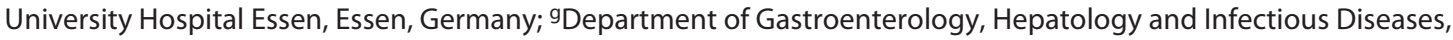

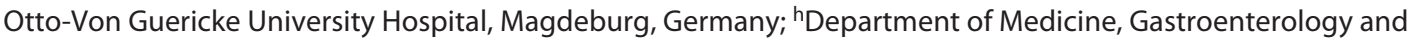
Hepatology, University Medical Center Hamburg-Eppendorf, Hamburg, Germany; 'Department of Internal Medicine 1, University Medical Center of the Johannes Gutenberg University Mainz, Mainz, Germany; ${ }^{j}$ Clinical Registry Unit (CRU), University Medical Center of the Johannes Gutenberg University Mainz, Mainz, Germany; ${ }^{\text {Department }}$ of Medicine I, University Medical Center Schleswig Holstein-Campus Lübeck, Lübeck, Germany; 'Department of Internal Medicine I, Ulm University Hospital, Ulm, Germany; 'mDepartment of Medicine II, Medical Center University

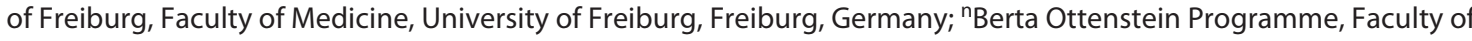
Medicine, University of Freiburg, Freiburg, Germany; ${ }^{\circ}$ Division of Hepatology, Department of Medicine II, University Hospital Würzburg, Würzburg, Germany; ${ }^{P}$ Department of Gastroenterology and Hepatology, Faculty of Medicine, University Hospital Cologne, University of Cologne, Cologne, Germany; 9Hepatology, Department of Clinical Research, University of Bern, Bern, Switzerland

\section{Keywords}

Hepatocellular carcinoma · Cabozantinib

\begin{abstract}
Background and Aims: The multikinase inhibitor cabozantinib has been approved for hepatocellular carcinoma (HCC) previously treated with sorafenib. We report safety and efficacy data of an international, multicenter, real-life cohort of patients with advanced HCC treated with cabozantinib. Methods: Patients with HCC who were treated with cabozantinib were retrospectively identified across 11 centers in
\end{abstract}

karger@karger.com www.karger.com/lic

Karger!"
(C) 2021 The Author(s)

Published by S. Karger AG, Basel

This is an Open Access article licensed under the Creative Commons Attribution-NonCommercial-4.0 International License (CC BY-NC) (http://www.karger.com/Services/OpenAccessLicense), applicable to the online version of the article only. Usage and distribution for commercial purposes requires written permission.
Austria, Switzerland, and Germany. Patients' characteristics, adverse events, duration of treatment and overall survival (OS) data were analyzed until April 1, 2020. Results: Eightyeight patients from 11 centers were included. The predominant underlying liver diseases were NAFLD/NASH in 26 (30\%) and hepatitis C infection in 21 (24\%) patients. Seventy-eight patients (89\%) were classified as Barcelona clinic liver cancer (BCLC) stage C. Sixty patients (68\%) were Child-Pugh A, whereas 22 (25\%) were Child-Pugh B, respectively. Cabozantinib was used as systemic second- and third-line or later treatment in $41(47 \%)$ and 46 (52\%) patients, respectively. The following best responses under cabozantinib were doc- 
umented: partial response in $6(7 \%)$, stable disease in 28 (32\%), and progressive disease in 28 (32\%) patients, respectively. Fifty-two patients (59\%) died during follow-up. The median OS from start of cabozantinib treatment was 7.0 months in the entire cohort and 9.7 months in Child-Pugh A patients, while Child-Pugh B patients had a median OS of 3.4 months, respectively. Thirty-seven (42\%) patients fulfilled the CELESTIAL inclusion and exclusion criteria, showing a median OS of 11.1 months. Most common adverse events were fatigue (15.6\%) and diarrhea (15.6\%). Conclusion: Cabozantinib treatment was effective, safe, and feasible in patients with advanced HCC in patients with compensated cirrhosis. Patients in the real-life setting had more advanced liver disease - in which $25 \%$ of patients were Child-Pugh B. However, OS in patients with Child-Pugh A cirrhosis was similar to that reported in the phase 3 trial (CELESTIAL).

(C) 2021 The Author(s) Published by S. Karger AG, Basel

\section{Introduction}

Hepatocellular carcinoma (HCC) is the most common malignant primary liver cancer and the third leading reason of cancer related death affecting more than half a million patients annually [1]. Curative HCC treatment is only available in early stages involving local ablative procedures, surgical resection, or liver transplantation. In patients not amenable to curative or local therapy as well as patients with metastatic disease, systemic treatment is the therapy of choice [2].

Since its approval in 2007, only sorafenib has been available as systemic treatment option for advanced HCC for several years [3]. Within the last 3 years the landscape of available treatment options has profoundly changed [4]. The TKI lenvatinib was noninferior to sorafenib in the first-line setting and the combination of the PD-L1 antibody atezolizumab and bevacizumab showed superiority to sorafenib and is considered the new standard of care also in Europe with the European Medical Agency (EMA) approval in November 2020 [5, 6]. Several drugs have been approved for second-line treatment of HCC by the United States Food and Drug Administration (FDA) and EMA including regorafenib, ramucirumab, and cabozantinib [7-10], whereas nivolumab, the combination of nivolumab and ipilimumab and pembrolizumab have only been approved by the FDA and not in Europe due to missing or negative phase 3 trial results [11-17].

In contrast to cabozantinib which has been approved for all sorafenib pretreated patients in the second- and third-line setting [18], ramucirumab use is restricted to patients with alpha-fetoprotein (AFP) levels $>400 \mathrm{ng} / \mathrm{mL}$ and regorafenib treatment is limited to sorafenib-tolerant patients. However, data of phase 3 trials are not always easily applicable in the real-life setting and patient cohorts differ significantly from those in everyday clinical practice. In all mentioned trials, only patients with wellcompensated cirrhosis (Child-Pugh A) were included. Notably, many patients treated in real life have more advanced liver disease, namely Child-Pugh B or even C. For these patients, data concerning efficacy of the indicated drugs are scarce. Only for sorafenib treatment, a large prospective registry is published, which reported similar efficacy of sorafenib in Child-Pugh A patients compared to the phase 3 trial. However, Child-Pugh B and C patients had a significant worse outcome [19]. A subgroup analysis from the registry showed no benefit for ramucirumab in comparison to placebo for Child-Pugh B patients [20]. For cabozantinib, no larger real-life data set has been reported. In the current article, we evaluated efficacy and safety of cabozantinib in a multicenter, international, real-life cohort of HCC patients.

\section{Patients and Methods}

Study Design and Selection of Patients

This was a retrospective study of patients treated with cabozantinib across 11 centers in Germany, Austria, and Switzerland. Patients with histologically or radiologically confirmed HCC who received cabozantinib as systemic treatment until April 2020 were eligible for inclusion into the analysis. Patients' data including history of the disease, treatment course, laboratory results, radiological data, and follow-up were collected retrospectively from patient files. The study was performed in accordance with the $1975 \mathrm{Dec}-$ laration of Helsinki. The retrospective analysis was approved by the local Ethics Committee (SGI03/18, Amendment 01/19) as well as the Ethics Committees of the individual centers.

\section{Assessments}

Electronic hospital charts were retrospectively analyzed for baseline demographic data and laboratory results. Radiological response was recorded by computed tomography (CT) or magnetic resonance imaging (MRI) at baseline, 6-12 weeks after treatment initiation, and about every $2-3$ months thereafter according to the local guidelines. Tumor response was assessed according to the Response Evaluation Criteria in Solid Tumors (RECIST) V1.1 [21] or modified RECIST [22] (according to centers preference). Side effects were recorded at every visit and graded according to the Common Terminology Criteria for Adverse Events (CTCAE) version 4.0 [23].

\section{Cabozantinib}

Cabozantinib is approved by the EMA, the Swissmedic from Switzerland, and the FDA for the treatment of patients with HCC who have been previously treated with sorafenib. The recom- 
Table 1. Patient characteristics

\begin{tabular}{|c|c|}
\hline Parameter & Patients \\
\hline \multicolumn{2}{|l|}{ Epidemiology } \\
\hline Patients, $n$ & 88 \\
\hline Gender, m/f (\%) & $76(86.4) / 12(13.6)$ \\
\hline Age, median, range & $65.6(25-84)$ \\
\hline \multicolumn{2}{|l|}{ Etiology of liver disease } \\
\hline Alcohol, $n(\%)$ & $18(20.5)$ \\
\hline Hepatitis C, $n(\%)$ & $21(23.9)$ \\
\hline Hepatitis B, $n(\%)$ & $7(8.0)$ \\
\hline $\mathrm{NASH}, n(\%)$ & $26(29.5)$ \\
\hline Cryptogenic/unknown, $n(\%)$ & $16(18.1)$ \\
\hline \multicolumn{2}{|l|}{ BCLC stage } \\
\hline $\mathrm{A}, n(\%)$ & $1(1.1)$ \\
\hline $\mathrm{B}, n(\%)$ & $8(9.1)$ \\
\hline C, $n(\%)$ & $78(88.6)$ \\
\hline $\mathrm{D}, n(\%)$ & $1(1.1)$ \\
\hline MVI, $n(\%)$ & $32(36.4)$ \\
\hline $\mathrm{EHS}, n(\%)$ & $60(68.2)$ \\
\hline \multicolumn{2}{|l|}{ Child-Pugh score } \\
\hline $\mathrm{A}, n(\%)$ & $60(68.2)$ \\
\hline $\mathrm{B}, n(\%)$ & $22(25.0)$ \\
\hline $\mathrm{C}, n(\%)$ & $1(1.1)$ \\
\hline \multicolumn{2}{|l|}{ ALBI grade } \\
\hline $1, n(\%)$ & $23(26.1)$ \\
\hline $2, n(\%)$ & $60(68.2)$ \\
\hline $3, n(\%)$ & $4(4.5)$ \\
\hline MELD, median, range & $9(6-20)$ \\
\hline \multicolumn{2}{|l|}{ ECOG } \\
\hline $0, n(\%)$ & $16(18.2)$ \\
\hline $1, n(\%)$ & $61(69.3)$ \\
\hline $2, n(\%)$ & $10(11.4)$ \\
\hline \multicolumn{2}{|l|}{ Prior treatment } \\
\hline Resection, $n(\%)$ & $34(38.6)$ \\
\hline Local ablation, ${ }^{*} n(\%)$ & $13(14.8)$ \\
\hline Loco-regional (TACE/SIRT), $n(\%)$ & $37(42.0) / 29(33.0)$ \\
\hline Liver transplantation, $n(\%)$ & $3(3.4)$ \\
\hline Previous sorafenib, $n(\%)$ & $81(92.0)$ \\
\hline \multicolumn{2}{|l|}{ Previous therapy lines, $n(\%)$} \\
\hline 1 & $41(46.6)$ \\
\hline 2 & $28(31.8)$ \\
\hline 3 & $15(17.0)$ \\
\hline 4 & $3(3.4)$ \\
\hline \multicolumn{2}{|l|}{ Laboratory results } \\
\hline BMI, median, range & $23.5(14-42)$ \\
\hline $\mathrm{ALT}, \mathrm{U} / \mathrm{L}$, median, range & $47(12-498)$ \\
\hline AST, U/L, median, range & $80.5(22-544)$ \\
\hline Bilirubin, $\mathrm{mg} / \mathrm{dL}$, median, range & $0.7(0.2-4.8)$ \\
\hline Albumin, $\mathrm{mg} / \mathrm{dL}$, median, range & $3.6(1.9-5.1)$ \\
\hline INR, mean, median, range & $1.12(0.9-2.8)$ \\
\hline Creatinine, $\mathrm{mg} / \mathrm{dL}$, median, range & $0.89(0.3-1.9)$ \\
\hline $\mathrm{CRP}, \mathrm{mg} / \mathrm{dL}$, median, range & $2.1(0.2-16.4)$ \\
\hline AFP, ng/mL, median, range & $98(0-127,135)$ \\
\hline $\mathrm{AFP}>400 \mathrm{ng} / \mathrm{mL}, n(\%)$ & $34(38.6)$ \\
\hline
\end{tabular}

NASH, nonalcoholic steatohepatitis; BCLC, Barcelona clinic liver cancer; MVI, macrovascular invasion; EHS, extrahepatic spread; MELD, model of end stage liver disease; ECOG, performance status Eastern Conference Oncology Group; TACE/SIRT, transarterial chemoembolization/selective internal radiotherapy; BMI, body mass index;ALT, alanineaminotransferase, AST, aspartateaminotransferase; INR, internationalized ratio; CRP, C-reactive protein; AFP, alphafetoprotein; ALBI, albumin-bilirubin; RFA, radiofrequency ablation; MWA, microwave ablation. * Including RFA and MWA. mended dose for cabozantinib in HCC is $60 \mathrm{mg}$ orally once daily. Dose delays or reductions were made based on toxicity and were suggested as 40 or $20 \mathrm{mg}$ as given in the summary of product characteristics.

\section{Statistical Analysis}

This study was designed as a retrospective cohort study. All patients were followed until death or last contact. The primary end point was overall survival (OS) and secondary end points included progression-free survival (PFS), response rate, and safety.

Data on baseline characteristics, radiological tumor response, and side effects were summarized using descriptive statistics. Continuous variables are shown as median and full range and categorical variables are reported as frequencies and percentages. Median duration of treatment was defined as time from the date of the first administration until the date of last documented administration. Patients still receiving cabozantinib at data cutoff were censored. Patients with at least one follow-up imaging assessment were evaluable for radiological response.

Data from patients, who died without radiologically confirmed tumor progression, were censored at the date of last radiological assessment or death. PFS was defined as the time from the date of first cabozantinib administration until radiological disease progression or death, whatever occurred first. Patients still alive and without radiologically confirmed progression at the date of last contact or data cutoff were censored. OS was defined as the time from start of cabozantinib until the date of death. Survival curves were calculated using the Kaplan-Meier method and compared by means of the log rank test. To analyze prognostic parameters a uniand multivariate Cox regression model with forward stepwise likelihood ratio was performed. Statistical analyses were performed with SPSS (Version 27.0, IBM, New York, NY, USA) and GraphPad Prism 8.0 (GraphPad Software, La Jolla, CA, USA). Differences between different patient cohorts were determined using the nonparametric Wilcoxon-Mann-Whitney and Kruskal-Wallis tests or Fisher's exact text. For subanalysis of multiple comparisons, the Bonferroni correction was used. $p$ values $<0.05$ were considered to be significant.

\section{Results}

\section{Patients}

Eighty-eight patients from 11 centers (1 Austrian center, 1 Swiss center, and 9 German centers) were included. To avoid a selection bias, we included every consecutive patient treated with cabozantinib retrospectively in the individual centers, no patients were excluded from analysis.

Individual patients had started cabozantinib treatment in the time from 16 July 2015 to 22 February 2020. Data cutoff for the analysis was 7th April 2020. Seventysix patients (86\%) were male and the median age was 66 years (range 25-84 years). Main baseline characteristics are shown in Table 1 . Most patients $(n=60,68 \%)$ were Child-Pugh stage A, whereas 22 (25\%) were Child-Pugh 
Fig. 1. a, b Kaplan-Meier analysis of OS and PFS. OS was defined as the time from starting cabozantinib to death from any cause and PFS as the time from starting cabozantinib to radiographic progression or death from any cause. Tick marks indicate censored data. OS, overall survival; PFS, progression-free survival; CI, confidence interval. a

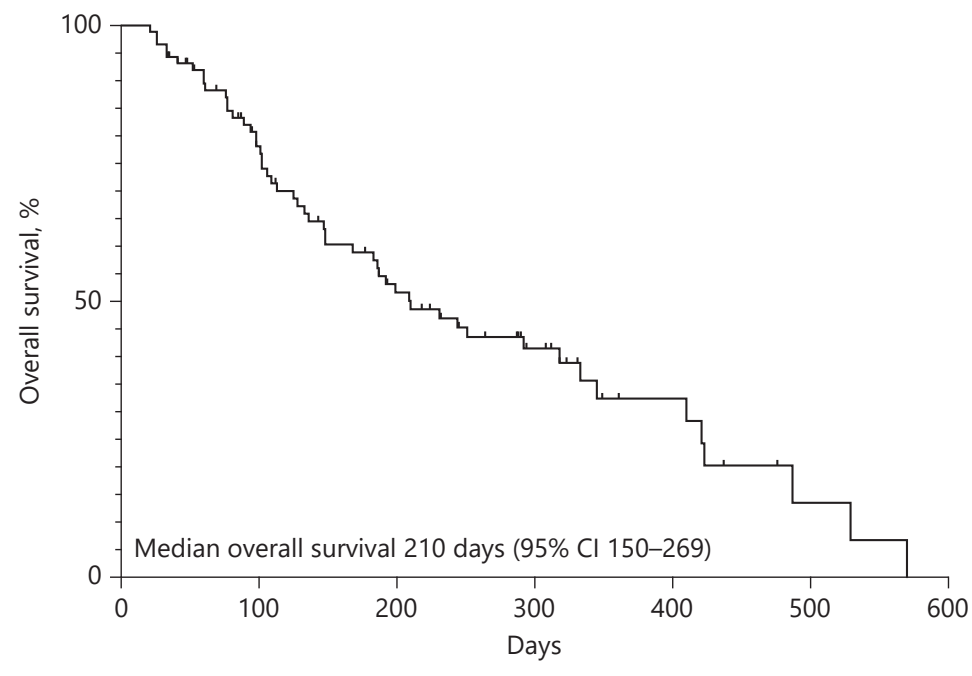

Patients at risk 88

62

35

20

9

2

1

b

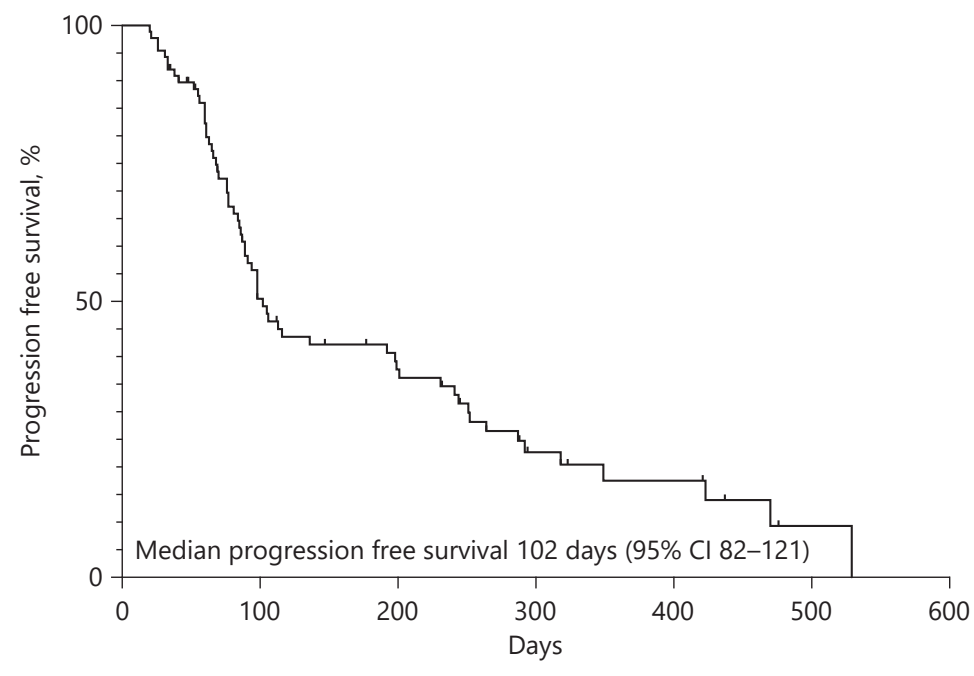

Patients at risk 88

26

11

2 stage B and one patient (1\%) was Child-Pugh stage C, in the remaining 5 patients Child-Pugh stage was not reported.

Eighty-one patients (92\%) had received sorafenib before cabozantinib treatment (median treatment time 287 days, range 8-2,320 days). Twenty-eight patients (32\%) had obtained 2 systemic therapy lines before initiation of cabozantinib and $15(17 \%)$ patients had 3 previous therapy lines, respectively. Three patients (3\%) even had 4 previous therapy lines. Twenty-five patients received at least one additional systemic anticancer drug following cessation of cabozantinib treatment. Fifty-two patients (59\%) died during follow-up. Median follow-up time af- ter start of cabozantinib was 4.8 months with a range of 0.7-18.4 months.

\section{Efficacy}

At last contact, 69 patients (78\%) had stopped cabozantinib treatment due to any form of disease progression as described above. Thirteen patients (15\%) were still on treatment and 6 patients (7\%) were lost to follow-up. Median time on cabozantinib was 69 days with a range of 3-437 days.

Overall, 62 patients had at least one follow-up imaging and assessment of tumor response was feasible. In 26 patients (30\%) response could not be assessed. Eighteen 
Table 2. Radiological response and survival data

\begin{tabular}{ll}
\hline Parameter & Patients \\
\hline Best documented response & \\
CR, $n(\%)$ & 0 \\
PR, $n(\%)$ & $6(6.8)$ \\
SD, $n(\%)$ & $28(31.8)$ \\
PD, $n(\%)$ & $28(31.8)$ \\
NA, $n(\%)$ & $26(29.5)$ \\
DCR, \% & 38.6 \\
PFS, median days (95\% CI), month & $102(82-121), 3.2$ months \\
OS, median days (95\% CI), month & $210(150-269), 6.8$ months \\
\hline
\end{tabular}

PFS, progression-free survival; OS, overall survival; CR, complete response; $\mathrm{PR}$, partial response; $\mathrm{SD}$, stable disease; $\mathrm{PD}$, progressive disease; NA, not evaluable; DCR, disease control rate.

(20\%) patients died before the first staging examination could be performed. These patients had a median OS of 51 days with a range of 21-89 days. Of the 18 patients, 16 were male (89\%), the etiology of liver disease was mainly NAFLD/NASH $(n=7,39 \%)$, and $15(83 \%)$ of the indicated patients had underwent prior sorafenib therapy. Seven of the 18 patients (39\%) were Child-Pugh B and 8 (44\%) patients had an AFP level above $400 \mathrm{ng} / \mathrm{mL}$. In these patients, median duration of cabozantinib treatment was 48 days with a range of 8-92 days. In 4 patients the treatment regimen was changed before first tumor assessment could be performed, in 3 patients cabozantinib was stopped on patients wish due to diarrhea grade 3 in one case and fatigue grade 2 in 2 cases, one patient had stopped without further documented reasons, and 4 patients were lost to follow-up.

None of the assessed patients showed a complete response (CR), 6 patients (7\%) had a partial response (PR), 28 patients (32\%) showed stable disease, and 28 patients (32\%) had progressive disease (PD), respectively. Taken together, we found an overall response rate (ORR) of 7\% and a disease control rate (DCR) of 39\%. Based on the entire cohort, the median PFS was 102 days (3.4 months) (95\% CI 82-121 days) and the OS after start of cabozantinib was 210 days (7.0 months) (95\% CI 150-269 days) as depicted in Figure 1a and b and Table 2.

Patients with only one prior systemic treatment line tended to have a longer median OS (244 days, 95\% CI 96-392 days) (8.1 months) than patients with 2 or more systemic treatment regimens before initiation of cabozantinib (209 days, 95\% CI 140-278 days) (7.0 months). However, the difference was not statistically significant $(p=0.88)$ (for treatment regimen before and after cabo- zantinib, see online suppl. material; for all online suppl. material, see www.karger.com/doi/10.1159/000515490).

OS did not differ among patients who received previous treatment with sorafenib compared to patients without sorafenib therapy before initiation of cabozantinib $(p=0.277)$. Additionally, no differences in OS were found between patients with or without a history of locoregional therapies in general $(p=0.637)$ and TACE $(p=0.590)$ or radioembolization $(p=0.575)$ in particular.

By a close margin, there was no statistically significant difference in OS between patients with AFP levels $>400$ $\mathrm{mg} / \mathrm{nL}$ compared to patients with low AFP levels $(p=$ 0.054 ). However, median OS was shorter (147 days, $95 \%$ CI 81-213 days) (4.9 months) in patients with AFP levels $>400 \mathrm{mg} / \mathrm{nL}$ compared to patients with lower AFP levels (292 days, 95\% CI 157-427 days) (9.7 months).

Patients with Barcelona clinic liver cancer (BCLC) C stage had a median OS of 199 days (95\% CI 135-263 days) (6.6 months) compared to patients with less advanced disease (BCLC B and BCLC A), who had a median survival of 423 days (95\% CI 232-614 days) (14.1 months), respectively $(p=0.056)$. The median OS for patients with PR or SD(stable disease) was 423 days (95\% CI 264-582 days) (14.1 months) and significantly longer compared to patients with PD (187 days; 95\% CI of 148-226 days) (6.2 months) ( $p=0.007$ ) as best response, respectively (Fig. 2).

We evaluated the whole cohort for patients fulfilling the CELESTIAL inclusion and exclusion criteria and included the patients whose information was fully available. We identified 37 (42\%) patients. The patients fulfilling the CELESTIAL inclusion criteria had a median OS of 333 days (95\% CI 224-441) compared to the patients who did not fulfill the CELESTIAL inclusion criteria who had a median OS of 192 days (95\% CI 97-286). The difference among the 2 cohort was not statistically significant, but showed a clear trend and was not significant in this cohort most probably due to small sample size (11.1 vs. 6.4 months, $p=0.06)$. PFS did not significantly differ among the 2 groups ( 106 days [ $95 \%$ CI $56-155$ ] vs. 98 days [ $95 \%$ CI 82-114], indicating 3.5 vs. 3.2 months, $p=0.4339$ ).

\section{Safety}

Recommended starting dose of cabozantinib in patients with HCC is $60 \mathrm{mg}$. In our cohort, 37 patients (42\%) received $60 \mathrm{mg}$ of cabozantinib, whereas 23 patients (26\%) started with $40 \mathrm{mg}$ and 28 patients (32\%) had 20 $\mathrm{mg}$ as initial dosage. Thirty-four patients (39\%) had dose reductions during treatment course. In 54 (61\%) patients, no reductions in dosing were reported. The reduced doses were $20 \mathrm{mg}$ in 17 patients (19\%) and $40 \mathrm{mg}$ in $15 \mathrm{pa}$ - 
Fig. 2. Kaplan-Meier analysis of OS according to response. OS was defined as the time from starting cabozantinib to death from any cause. $\mathrm{PR}+\mathrm{SD}$ are patients with partial response or stable disease compared to patients with PD. Curve comparison by log rank test. Tick marks indicate censored data. OS, overall survival; $\mathrm{PD}$, progressive disease; PR, partial response; CI, confidence interval; $\mathrm{HR}$, hazard ratio; $\mathrm{SD}$, stable disease.

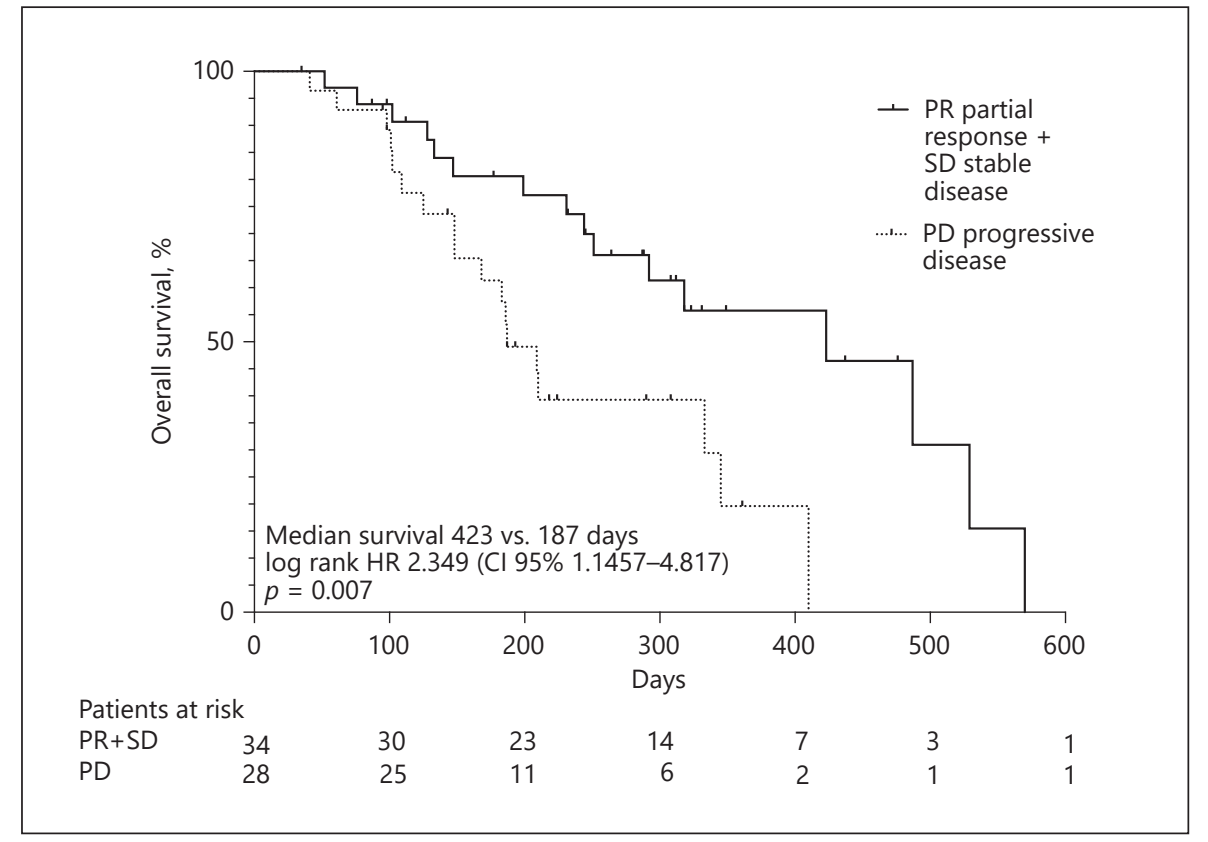

Table 3. Documented adverse events

\begin{tabular}{lcl}
\hline & $\begin{array}{l}\text { Any grade, } \\
n(\%)\end{array}$ & $\begin{array}{l}\geq \text { Grade 3, } \\
n(\%)\end{array}$ \\
\hline Diarrhea & $15(16.5)$ & $8(8.8)$ \\
Fatigue & $15(16.5)$ & $4(4.4)$ \\
Loss of appetite & $10(11)$ & $1(1.1)$ \\
Nausea & $6(6.6)$ & $3(3.3)$ \\
Palmar-plantar erythrodysesthesia & $6(6.6)$ & $1(1.1)$ \\
Vomiting & $3(3.3)$ & \\
Rash & $3(3.3)$ & \\
Hypertension & $2(2.2)$ & $1(1.1)$ \\
Weight loss & $2(2.2)$ & \\
Hypocalcemia & $1(1.1)$ & $1(1.1)$ \\
Nephrotic syndrome & $1(1.1)$ & $1(1.1)$ \\
Stomatitis & $1(1.1)$ & $1(1.1)$ \\
Vertigo & $1(1.1)$ & \\
Bone pain & $1(1.1)$ & \\
Hyperbilirubinemia & $1(1.1)$ & \\
Sore mouth & $1(1.1)$ & \\
Abdominal pain & $1(1.1)$ & \\
Cramps & $1(1.1)$ & $1(1.1)$ \\
Leucocytoclastic vasculitis & $1(1.1)$ & \\
\hline
\end{tabular}

tients (17\%). In 21 patients (24\%) dose escalation was reported during treatment. In 45 patients (51\%) at least one adverse event (AE) was reported. Four patients stopped cabozantinib before the first staging, all on patients' wish. One patient developed diarrhea grade 3 and 2 patients developed fatigue grade 2 . The incidence of the individual AEs is shown in Table 3.

We further analyzed the 28 patients who started with $20 \mathrm{mg}$ and compared these patients to the rest of the cohort. We found a comparable adverse event rate (55 vs. $48 \%$ ) and no difference in ECOG, etiology of liver disease or Child-Pugh stage distribution, respectively. However, patients with $20 \mathrm{mg}$ as a starting dose had significantly higher likeliness to be in a later treatment line ( $>2$ previous treatment lines $n=21$ [75\%] in the $20 \mathrm{mg}$ group vs. $n=26[43 \%]$ in the remaining cohort, $p=0.006$ ).

\section{Efficacy and Safety according to Child-Pugh Stage}

In real life, many patients at need for systemic treatment have more advanced cirrhosis and are formally classified as Child-Pugh stage B. However, these patients were excluded in the CELESTIAL trial, a common practice in HCC trials [8]. Therefore, safety and efficacy data in patients with impaired liver function is desirable. In this cohort, most patients $(n=60,68 \%)$ were Child-Pugh stage A. However, 22 (25\%) were Child-Pugh stage B and one patient (1\%) was Child-Pugh stage C.

We compared PFS and OS among patients with compensated liver disease (Child-Pugh A) and more advanced disease, mainly Child-Pugh B patients. Patients stratified for Child-Pugh A cirrhosis had a median OS of 292 days (95\% CI 171-429 days) (9.7 months). Patients with stage Child-Pugh B7 only showed a median OS of 
Fig. 3. Kaplan-Meier analysis of OS according to Child-Pugh stage. OS was defined as the time from starting cabozantinib to death from any cause. Comparison of patients with Child-Pugh stage A versus Child-Pugh stage B. Curve comparison by log rank test. Tick marks indicate censored data. OS, overall survival; CI, confidence interval; HR, hazard ratio.

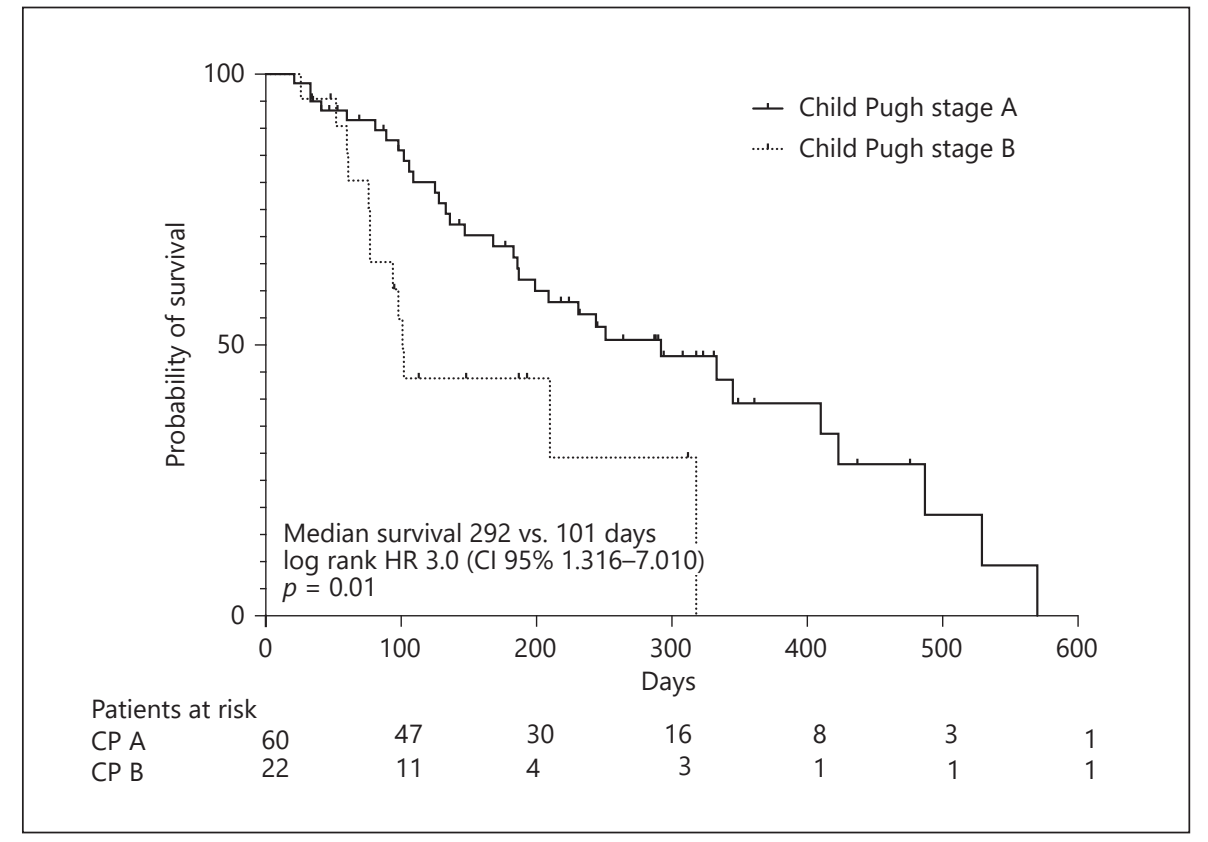

Table 4. Univariate and multivariate analyses of parameters associated with OS

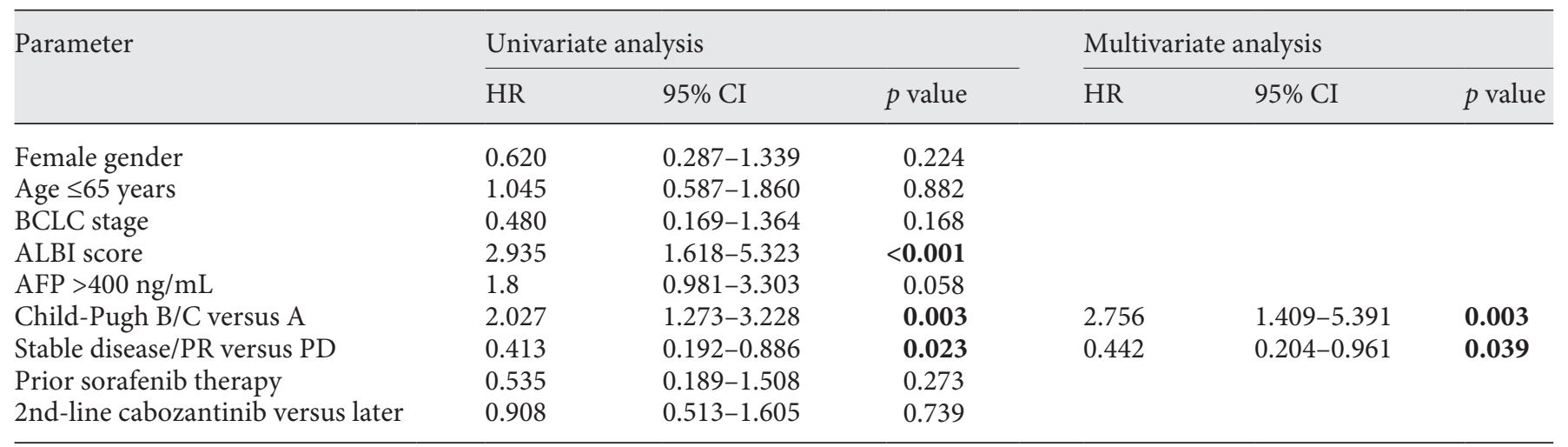

CI, confidence interval; HR, hazard ratio; BCLC, Barcelona clinic liver cancer; ALBI score, albumin-bilirubin score; AFP, alphafetoprotein; PR, partial response; PD, progressive disease; OS, overall survival. Values in bold signify $p<0.05$.

210 days (95\% CI 52-368 days) (7.0 months). In contrast, patients with a more advanced liver disease (Child-Pugh B8, B9, and C) had a much less favorable median OS of 101 days (95\% CI of 73-129 days) (3.4 months). The difference concerning OS was statistically significant $(p<$ $0.001)$. Figure 3. PFS was median 98 days (79-116) in Child-Pugh A and median 94 days (73-114) in ChildPugh B.

Safety analysis showed 26 documented AEs in ChildPugh A patients (43.3\%) compared to 16 in Child-Pugh
B patients (72.7\%), which was significantly different $(p=$ 0.017 ). From the 4 patients who stopped cabozantinib treatment early due to an AE, 2 were Child-Pugh B. In the Child A cohort, 33 patients out of 60 (55\%) started with reduced dose ( 20 or $40 \mathrm{mg}$ ) and in the Child B cohort, 18 out of 22 patients $(81.8 \%)(p=0.04)$. However, during treatment, there was no significant difference in patients with dose reduction ( 40 vs. $36.3 \%, p=0.84$ ). 


\section{Factors Associated with Survival}

Known predictors of survival in patients with HCC include liver function and AFP levels. The variables gender, age ( $\leq 65$ years vs. $>65$ years) [24], the BCLC stage, AFP levels ( $\leq 400 \mathrm{ng} / \mathrm{mL}$ vs. $>400 \mathrm{ng} / \mathrm{mL}$ ) [25], the albuminbilirubin (ALBI) score [26], the treatment response, prior sorafenib therapy, and the treatment line [27] were included in a univariate cox regression model. Furthermore, all factors from univariate analysis with a $p$ value $<0.1$ were included in the multivariate model. As shown in Table 4, only the Child-Pugh stage and the treatment response were independently associated with OS.

\section{Discussion}

Cabozantinib has been the first drug for second-line and third-line treatment of sorafenib pretreated HCC patients. Furthermore, following first-line systemic treatment cabozantinib is the only drug in this setting which can be prescribed to all patients irrespective of baseline AFP levels or tolerability of sorafenib treatment. This differentiates it from ramucirumab and regorafenib $[7,8$, 28]. Especially in the EMA area in Europe, where nivolum$\mathrm{ab}$ and pembrolizumab have not been approved for HCC due to negative phase 3 trials [11,29], it is at least formally the standard drug for sorafenib pretreated HCC patients.

It is well known that patients in real-life more often have advanced liver disease than patients included in interventional trials. In our cohort of patients from several centers in Europe, we found a substantial number of patients with more advanced liver disease, namely ChildPugh B patients, who were treated with cabozantinib. More advanced liver disease is associated with a poorer overall prognosis and also the benefit from systemic treatment with TKIs such as sorafenib and the VEGFR2 directed antibody ramucirumab seem to be much less in patients with Child-Pugh B and C cirrhosis compared to Child-Pugh A cirrhosis $[19,20]$. In our cohort, patients with Child-Pugh B7 had a worse outcome than ChildPugh A patients, but a more favorable prognosis than patients with more advanced disease, indicating that these patients still benefit from systemic treatment. On the other hand, patients with more advanced cirrhosis should not receive cabozantinib as recommend by current guidelines [2]. Similar to sorafenib, hepatobiliary elimination appears to be the major route of elimination of cabozantinib and its metabolites. Although metabolized, cabozantinib appears to be the pharmacologically active circu- lating analyte [30]. Data on cabozantinib in patients with impaired renal or hepatic function are scarce; pharmacokinetics studies were done for single dose $60 \mathrm{mg}$ /daily. Cabozantinib plasma exposures were minimally increased $(<30 \%)$ in subjects with mild- or moderate-renal impairment compared to those with normal-renal function. Exposures to cabozantinib in subjects with mild and moderate hepatic impairment were moderately increased compared to subjects with normal-hepatic-function [31].

As mild to moderate hepatic impairment nearly doubles exposure rates, severe hepatic impairment (i.e., Child-Pugh B and C cirrhosis) could profoundly hamper the drug metabolism leading to even much higher drug concentrations and potentially more adverse effects. Recently, El-Khoueiry and colleagues [32] published an abstract of a subgroup analysis of patients from CELESTIAL who deteriorated to Child-Pugh B 8 weeks after starting cabozantinib but still showed an OS of 8.5 months. Considering our patients with Child-Pugh B7 with an OS of 7 months, this is comparable, especially considered that at study entry, $75 \%$ of patients had Child-Pugh A6 and 25\% had A5 in CELESTIAL.

The OS in our cohort in the subset of patients with only Child-Pugh A cirrhosis was comparable to the phase 3 CELESTIAL trial with a median OS of 9.7 months. Moreover, patients in our cohort who formally fulfilled the inclusion criteria for the CELESTIAL trial had a median OS of 11.1 months which is even longer than the median OS in the CELESTIAL registry trial of 10.2 months [8].

Patients with higher AFP levels who have generally a poorer prognosis tended to have a shorter median OS under treatment with cabozantinib compared to patients with lower AFP levels. In our cohort, prognosis of patients with or without previous locoregional pretreatment did not differ, which is in line with the CELESTIAL trial, where efficacy of cabozantinib was also irrespective of pretreatment with TACE [33]. Recommended cabozantinib starting dose is $60 \mathrm{mg}$ daily. In the CELESTIAL trial, the average cabozantinib dose administered to the patients was $38.5 \mathrm{mg} /$ days [8]. In our patients $<50 \%$ received $60 \mathrm{mg}$ as starting dose. The other patients started with a daily dose of $40 \mathrm{mg}$ or even only $20 \mathrm{mg}$. Nevertheless, further dose reductions were done in nearly $40 \%$ of patients. Of note, in $>20 \%$ of patients drug dose was increased during treatment course. We could not find any difference in OS or PFS in the patients who started with lower cabozantinib dosage than patients who stated with the standard dose of $60 \mathrm{mg}$. As this was a retrospective clinical cohort, we only can speculate about the reasons 
for starting with lower doses; however, mainly the consideration would be to reduce side effects in the beginning to rise treatment adherence. The common practice to start with reduced doses has led to clinical trials such as the CABORISE trial which investigates planned dose escalation in HCC patients (NCT 04522908). AEs were also commonly reported in our patients and were completely comparable to AEs reported in CELESTIAL, however, much less frequent (e.g., diarrhea 54 vs. 17\%). We can only speculate about the reason, however, as documentation of adverse advents is mandatory in trials but not in real-life most probably not all adverse events in number were documented. However, there were no new safety signals in this study especially not in advanced liver disease patients.

Tovoli and colleagues [34] published an abstract evaluating 52 patients from different centers treated with cabozantinib in hepatocellular in real-life, which was the only data we found on this topic. They only included patients with compensated liver disease (e.g., only Child A patients) and found a median OS of 12.9 months with comparable efficacy and safety to the CELESTIAL trial. This is in accordance to our data and underlines the finding of good efficacy of cabozantinib in a real-world setting in patients with preserved liver function.

Several drugs have shown less effectiveness in HCC in real-life compared to trial data, especially in advanced stages of cirrhosis $[35,36]$, the main reason might be the narrow eligibility criteria in drug trials making the results not comparable to the HCC population. In this subset of patients in a real-life setting, cabozantinib showed comparable efficacy (OS beyond 9 months in Child-Pugh A patients) and safety in patients with preserved liver function and confirmed the reported findings of the CELESTIAL trial.

\section{Statement of Ethics}

The research was conducted ethically in accordance with the World Medical Association Declaration of Helsinki. As a retrospective study, written informed consent was not needed and waived by the Ethics Committee (Frankfurt/Main (SGI03/18, Amendment 01/19). The study protocol was fully approved.

\section{Conflict of Interest Statement}

F.F. received travel support from Abbvie and Ipsen and speaker fees from AbbVie. B.S. received travel support from AbbVie, Gilead, and Ipsen. C.L. reports no conflicts of interest. J.B. served as Consultant for BTGPLC, BMS, MSD, Roche, and Eisai. He served as a Speaker for BTGPLC, Eisai, and Novartis. He received travel support from Ipsen, BTGPLC, and BMS. He is an investigator for MSD, BMS, Lilly. T.W.F. reports no conflicts of interest. C.C. received fees and travel support from Eisai, MSD, Ipsen, and Falk Foundation. A.B. reports no conflicts of interest. D.B. served as a consultant for Bayer Healthcare, Boston Scientific, and Falk Foundation. J.W. served as a consultant/speaker and received travel support from BMS, Eisai, Falk, Gilead, Ipsen, MSD, and Novartis. He is an investigator for MSD. T.M. reports no conflicts of interest. F.K. has received speakers' fees from Bayer, Ipsen, MSD, Eisai, Shire, Sirtex and has received travel grants from Eisai, Janssen, Ipsen, and Novartis. D.T.W. reports no conflicts of interest. M.S. served as a consult for Bayer Healthcare, and Falk Foundation. T.J.E: served as a speaker and/or consultant for AstraZeneca, Bayer, BMS, Celgene, Eisai, Incyte, Ipsen, Merck Serono, MSD, Roche, and Servier. He received travel support from Ipsen. He received financial support for scientific projects from Servier. A.W. is an Editorial Board Member of Liver Cancer. H.W. served as speaker for Bayer, Eisai, and Ipsen, and as a consultant for Bayer, Eisai, Lilly, BMS, Roche, and Ipsen. He conducts studies for Bayer, Roche, Lilly, MSD, and BMS. M.V. received honoraria from Merck Serono, Bayer Vital, and Sirtex and is a member of the advisory boards of Ipsen, Roche, Bayer, Lilly, Nordic Pharma, BMS, MSD, and Amgen. C.L. served as a speaker and/or consultant for AbbVie, Gilead, MSD, Norgine, Falk, Eisai, Roche, Behring, and travel received support from AbbVie and Gilead. M.P. is an investigator for Bayer, BMS, Lilly, and Roche; he received speaker honoraria from Bayer, BMS, Eisai, Lilly, and MSD; he is a consultant for Bayer, BMS, Ipsen, Eisai, Lilly, MSD, and Roche; he received travel support from Bayer and BMS. J-F.D. served in advisory committees: Abbvie, Bayer, Bristol-Myers Squibb, Falk, Genfit, Genkyotex, Gilead Sciences, HepaRegenix, Intercept, Lilly, Merck, and Novartis. Speaking and teaching: Bayer, Bristol-Myers Squibb, Intercept, Genfit, Gilead Sciences, Novartis, and Roche. O.W. served as a speaker and/or consultant for AstraZeneca, Amgen, Bayer, BMS, Celgene, Eisai, Incyte, Ipsen, Merck Serono, MSD, Novartis, Roche, Servier, and Shire. He received travel support from Abbvie, Bayer, BMS, Gilead, Ipsen, Medac, and Merck. He received financial support for scientific projects from Else Kröner-FreseniusStiftung, IPSEN, Medac, Merck Serono, and Novartis. He is an investigator for Basilea, Incyte, and MSD.

\section{Funding Sources}

The authors received no specific funding for this work.

\section{Author Contributions}

F.F., B.S., C.L., J.B., T.W.F., C.C., A.B., D.B., J.W., T.M., F.K., D.T.W., P.R., M.S., K.H.P., T.J.E., A.W., H.W., M.V., J.F.D., C.M.L., M.P., and O.W. collected and analyzed the data. F.F. and O.W. designed the research study and wrote the manuscript. All authors approved the final version of the manuscript. 


\section{References}

1 Villanueva A. Hepatocellular carcinoma. N Engl J Med. 2019;380(15):1450-62.

2 Galle PR, Forner A, Llovet JM, Mazzaferro V, Piscaglia F, Raoul J-L, et al. EASL clinical practice guidelines: management of hepatocellular carcinoma. J Hepatol. 2018;69(1):182-236.

3 Llovet JM, Ricci S, Mazzaferro V, Hilgard P, Gane E, Blanc JF, et al. Sorafenib in advanced hepatocellular carcinoma. $\mathrm{N}$ Engl J Med. 2008;359(4):378-90.

4 von Felden J. New systemic agents for hepatocellular carcinoma: an update 2020. Curr Opin Gastroenterol. 2020;36(3):177-83.

5 Kudo M, Finn RS, Qin S, Han K-H, Ikeda K, Piscaglia F, et al. Lenvatinib versus sorafenib in first-line treatment of patients with unresectable hepatocellular carcinoma: a randomised phase 3 non-inferiority trial. Lancet. 2018;391(10126):1163-73.

6 Finn RS, Qin S, Ikeda M, Galle PR, Ducreux M, Kim TY, et al. Atezolizumab plus bevacizumab in unresectable hepatocellular carcinoma. N Engl J Med. 2020;382(20):1894-905.

7 Bruix J, Qin S, Merle P, Granito A, Huang YH, Bodoky G, et al. Regorafenib for patients with hepatocellular carcinoma who progressed on sorafenib treatment (RESORCE): a randomised, double-blind, placebo-controlled, phase 3 trial. Lancet. 2017;389(10064):56-66.

8 Abou-Alfa GK, Meyer T, Cheng AL, ElKhoueiry AB, Rimassa L, Ryoo BY, et al. Cabozantinib in patients with advanced and progressing hepatocellular carcinoma. N Engl J Med. 2018;379(1):54-63.

9 Zhu AX, Kang YK, Yen CJ, Finn RS, Galle PR, Llovet JM, et al. Ramucirumab after sorafenib in patients with advanced hepatocellular carcinoma and increased $\alpha$-fetoprotein concentrations (REACH-2): a randomised, doubleblind, placebo-controlled, phase 3 trial. Lancet Oncol. 2019;20:282-96.

10 Rimassa L, Pressiani T, Merle P. Systemic treatment options in hepatocellular carcinoma. Liver Cancer. 2019;8(6):427-46.

11 Yau T, Park JW, Finn RS, Cheng A-L, Mathurin P, Edeline J, et al. CheckMate 459: a randomized, multi-center phase III study of nivolumab (NIVO) vs sorafenib (SOR) as first-line (1L) treatment in patients (pts) with advanced hepatocellular carcinoma (aHCC). Ann Oncol. 2019;30:v874-5.

12 Yau T, Kang YK, Kim TY, El-Khoueiry AB, Santoro A, Sangro B, et al. Efficacy and safety of nivolumab plus ipilimumab in patients with advanced hepatocellular carcinoma previously treated with sorafenib: the CheckMate 040 randomized clinical trial. JAMA Oncol. 2020;6(11):e204564.

13 El-Khoueiry AB, Sangro B, Yau T, Crocenzi TS, Kudo M, Hsu C, et al. Nivolumab in patients with advanced hepatocellular carcinoma (CheckMate 040): an open-label, noncomparative, phase $1 / 2$ dose escalation and expansion trial. Lancet. 2017;389(10088): 2492-502.
14 Zhu AX, Finn RS, Edeline J, Cattan S, Ogasawara S, Palmer D, et al. Pembrolizumab in patients with advanced hepatocellular carcinoma previously treated with sorafenib (KEYNOTE-224): a non-randomised, openlabel phase 2 trial. Lancet Oncol. 2018;19: 940-52.

15 Finn RS, Ryoo BY, Merle P, Kudo M, Bouattour M, Lim HY, et al. Pembrolizumab as second-line therapy in patients with advanced hepatocellular carcinoma in KEYNOTE-240: a randomized, double-blind, phase III trial. J Clin Oncol. 2020;38(3):193-202.

16 Pinter M, Jain RK, Duda DG. The current landscape of immune checkpoint blockade in hepatocellular carcinoma a review. JAMA Oncol. 2021;7(1):113-23.

17 Pinter M, Scheiner B, Peck-Radosavljevic M. Immunotherapy for advanced hepatocellular carcinoma: a focus on special subgroups. Gut. 2021;70(1):204-14

18 Kudo M. Cabozantinib as a second-line agent in advanced hepatocellular carcinoma. Liver Cancer. 2018;7(2):123-33.

19 Marrero JA, Kudo M, Venook AP, Ye SL, Bronowicki JP, Chen XP, et al. Observational registry of sorafenib use in clinical practice across Child-Pugh subgroups: the GIDEON study. J Hepatol. 2016;65(6):1140-7.

20 Zhu AX, Baron AD, Malfertheiner P, Kudo M, Kawazoe S, Pezet D, et al. Ramucirumab as second-line treatment in patients with advanced hepatocellular carcinoma: analysis of REACH trial results by Child-Pugh score. JAMA Oncol. 2017;3(2):235-43.

21 Eisenhauer EA, Therasse P, Bogaerts J, Schwartz LH, Sargent D, Ford R, et al. New response evaluation criteria in solid tumours: revised RECIST guideline (version 1.1). Eur J Cancer. 2009;45(2):228-47.

22 Lencioni R, Llovet JM. Modified RECIST (mRECIST) assessment for hepatocellular carcinoma. Semin Liver Dis. 2010;30(1):5260.

23 Cancer Institute N. Common terminology criteria for adverse events (CTCAE) common terminology criteria for adverse events v4.0 (CTCAE). 2009. Available from: https://evs. n ci.nih.gov/ft p 1 / C T C A E / C T $\mathrm{CA} \mathrm{E} \_4.03 \ldots 2010-06-14$ _ QuickReference_5x7.pdf.

24 Rimassa L, Cicin I, Blanc J-F, Klümpen HJ, Zagonel V, Tran A, et al. Outcomes based on age in the phase 3 CELESTIAL trial of cabozantinib (C) versus placebo $(\mathrm{P})$ in patients (pts) with advanced hepatocellular carcinoma (HCC). J Clin Oncol. 2018;36(15_Suppl 1): 4090.

25 Kelley RK, Meyer T, Rimassa L, Merle P, Park JW, Yau T, et al. Serum alpha-fetoprotein levels and clinical outcomes in the phase III CELESTIAL Study of cabozantinib versus placebo in patients with advanced hepatocellular carcinoma. Clin Cancer Res. 2020;26(18): 4795-804.
26 Chan SL, Miksad R, Cicin I, Chen Y, Klumpen HJ, Kim S, et al. Outcomes based on albuminbilirubin (ALBI) grade in the phase III CELESTIAL trial of cabozantinib versus placebo in patients with advanced hepatocellular carcinoma (HCC). Ann Oncol. 2019;30:ix45-6.

27 Kelley RK, Ryoo BY, Merle P, Park JW, Bolondi L, Chan SL, et al. Second-line cabozantinib after sorafenib treatment for advanced hepatocellular carcinoma: a subgroup analysis of the phase 3 CELESTIAL trial. ESMO Open. 2020;5(4):1-9.

28 Zhu AX, Kang Y-K, Yen C-J, Finn RS, Galle PR, Llovet JM, et al. REACH-2: a randomized, double-blind, placebo-controlled phase 3 study of ramucirumab versus placebo as second-line treatment in patients with advanced hepatocellular carcinoma (HCC) and elevated baseline alpha-fetoprotein (AFP) following first-line sorafe. J Clin Oncol. 2018;36: 4003.

29 Finn RS, Chan SL, Zhu AX, Knox JJ, Cheng A-L, Siegel AB, et al. KEYNOTE-240: randomized phase III study of pembrolizumab versus best supportive care for second-line advanced hepatocellular carcinoma. J Clin Oncol. 2017;35(4_Suppl 1):TPS503.

30 Lacy SA, Miles DR, Nguyen LT. Clinical pharmacokinetics and pharmacodynamics of cabozantinib. Clin Pharmacokinet. 2017; 56(5):477-91.

31 Nguyen L, Holland J, Ramies D, Mamelok R, Benrimoh N, Ciric S, et al. Effect of renal and hepatic impairment on the pharmacokinetics of cabozantinib. J Clin Pharmacol. 2016; 56(9):1130-40.

32 El-Khoueiry A, Meyer T, Cheng A, Rimassa L, Sen S, Milwee S, et al. SO-9 outcomes for patients with advanced hepatocellular carcinoma and Child-Pugh B liver function in the phase 3 CELESTIAL study of cabozantinib vs placebo. Ann Oncol. 2020;31:S220.

33 Yau T, Cheng A-L, Meyer T, Ryoo B-Y, Park J-W, Klümpen H-J, et al. Outcomes by prior transarterial chemoembolization (TACE) in the phase III CELESTIAL trial of cabozantinib $(\mathrm{C})$ versus placebo $(\mathrm{P})$ in patients (pts) with advanced hepatocellular carcinoma (HCC). Ann Oncol. 2018;29:viii237-8.

34 Tovoli F, Dadduzio V, De Lorenzo S, Garajovà $\mathrm{I}$, Marra $\mathrm{F}$, Trevisani $\mathrm{F}$, et al. 999P reallife clinical data of cabozantinib for unresectable hepatocellular carcinoma. Ann Oncol. 2020;31:S695.

35 Sanoff HK, Chang Y, Lund JL, O’Neil BH, Dusetzina SB. Sorafenib effectiveness in advanced hepatocellular carcinoma. Oncologist. 2016;21(9):1113-20.

36 Ganten TM, Stauber RE, Schott E, Malfertheiner P, Buder R, Galle PR, et al. Sorafenib in patients with hepatocellular carcinoma-results of the observational INSIGHT study. Clin Cancer Res. 2017;23(19):5720-8. 